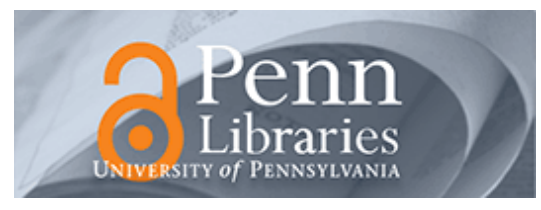

University of Pennsylvania ScholarlyCommons

\title{
Bridging Contested Terrain: Linking Incentive-Based and Learning Perspectives on Organizational Evolution
}

Giovanni Dosi

Daniel A. Levinthal

University of Pennsylvania

Luigi Marengo

Follow this and additional works at: https://repository.upenn.edu/mgmt_papers

Part of the Business Administration, Management, and Operations Commons

\section{Recommended Citation}

Dosi, G., Levinthal, D. A., \& Marengo, L. (2003). Bridging Contested Terrain: Linking Incentive-Based and Learning Perspectives on Organizational Evolution. Industrial and Corporate Change, 12 (2), 413-436. http://dx.doi.org/10.1093/icc/12.2.413 


\title{
Bridging Contested Terrain: Linking Incentive-Based and Learning Perspectives on Organizational Evolution
}

\author{
Abstract \\ In this paper we present a general model of organizational problem-solving in which we explore the \\ relationship between problem complexity, decentralization of tasks and reward schemes. When facing \\ complex problems that require the co-ordination of large numbers of interdependent elements, \\ organizations face a decomposition problem that has both cognitive dimensions and reward and \\ incentive dimensions. The former relate to the decomposition and allocation of the process of generation \\ of new solutions: since the search space is too vast to be searched extensively, organizations employ \\ heuristics for reducing it. The decomposition heuristic takes the form of division of cognitive labour and \\ determines which solutions are generated and become candidates for selection. The reward and \\ incentive dimensions fundamentally shape the selection environment which chooses over alternative \\ solutions. The model we present begins to study the interrelationships between these two domains of \\ analysis: in particular, we compare the problem-solving performance of organizations characterized by \\ various decompositions (of coarser or finer grain) and various reward schemes (at the level of the entire \\ organization, team and individual). Moreover we investigate extensions of our model in order to account \\ for (admittedly rudimentary) power and authority relationships (giving some parts of the organization the \\ power to stop changes in other parts), and discuss the interaction of problem representations and \\ incentive mechanisms.

\section{Disciplines} \\ Business Administration, Management, and Operations
}




\title{
BRIDGING CONTESTED TERRAIN: LINKING INCENTIVE-BASED AND Learning Perspectives on Organizational Evolution
}

\author{
Giovanni DOSI*, Daniel LEVINTHAL** and Luigi MARENGO***
}

May 20001

\section{PRELIMINARY DRAFT}

*St. Anna School of Advanced Studies, Pisa, Italy, gdosi@sssup.it

**Wharton School, University of Pennsylvania, Philadelphia, USA, levinthal@wharton.upenn.edu ***Dept. of Economics, University of Trento, Trento, Italy, Imarengo@gelso.unitn.it 


\begin{abstract}
In this paper we present a general model of organizational problem-solving in which we explore the relationship between problem complexity, decentralization of tasks and reward schemes. When facing complex problems which require the coordination of large numbers of interdependent elements, organization face a decomposition problem which has both a cognitive dimension and a reward and incentive dimension. The former relates to the decomposition and allocation of the process of generation of new solutions: since the search space is too vast to be searched extensively, organizations employ heuristics for reducing it. The decomposition heuristic takes the form of division of cognitive labor and determines which solutions are generated and become candidates for selection. The reward and incentive dimension defines the selection environment which chooses over alternative solutions.

The model we present studies the interrelationships between these two dimensions, in particular we compare the problem solving performance of organizations characterized by various decompositions (of coarser of finer grain) and various reward schemes (at the level of the entire organization, team and individual). Moreover we extend our model - in a still tentative fashion - in order to account for such power and authority relationships (giving some parts of the organization the power to stop changes in other parts), and to discuss the co-evolution of problem representations and incentive mechanisms.
\end{abstract}




\section{Introduction}

Social organizations - in their impressive variety over history, across societies and across domains of human activities - generally display also very diverse forms of division of operational and 'cognitive' labour, and, at the same time, equally diverse hierarchical arrangements, distributions of power, and mechanisms of elicitation of efforts by individual agents. While it is easy to see that this is generally the case, it is much more difficult to disentangle the different domains of analysis which tend to correspond to multiple, co-existing, levels of interactions amongst organizational members. These levels of interaction are also likely to map onto different forms of 'social embeddedness' of individual actions. In many respects, the understanding of these processes is one of the fundamental tasks of social sciences since their origins. The task is obviously enormous. It may clearly begin from different angles. It happens that the dominant strand of contemporary analyses - at least those regarding economic organizations - start with 'primitives' of the interpretation of the nature of organizations themselves building upon sophisticatedly, selfseeking, often forward looking, rather mean, agents. The "energies" of these self-interested and sophisticated actors is viewed as typically controlled and directed by market forces. In those settings in which, due to failures of information and contract completeness, markets are less effective in this task, then organizations are called for to surrogate such imperfections. It is a story too familiar to be repeated here. ${ }^{1}$

Conversely, a small - but not negligible and growing - minority of the economic profession has placed the (first approximation) "primitives" of the analysis of the nature of economic organizations in their problem-solving features, in turn nested in ubiquitous forms if human "bounded rationality", grossly imperfect processes of learning and diverse mechanisms of social distribution of "cognitive labor". Needless to say, it is a perspective which find seminal roots in the works of Herbert Simon, James March and indeed Dick Nelson and Sidney Winter.

We offer the following caricature to illustrate the differences between the two interpretative philosophies. Suppose that two delegations of intelligent but totally uninformed beings from Mars are sent to the Earth with the mandate of reporting what "what business firms are." The delegations are not allowed to visit the firms themselves. Rather, the first one is given to read, out of an enormous literature, say Tirole (1988), Grossman and Hart (1986), Laffont and Tirole (....), while the second is given Simon (1981), Nelson and Winter (1982), Radner (1986), Winter (1988) and maybe some works of ours - e.g. Dosi (1988), Levinthal (1997), Dosi and Marengo (1995), Gavetti and Levinthal (2000).

\footnotetext{
${ }^{1}$ A few more detailed epistemological remarks are provided in Dosi (1995) and Coriat and Dosi (1998b)
} 
What would they report back to Mars? (We reasonably assume that these entities, given their empirical naiveté, are unable to catch all the caveats from footnotes, side remarks, etc...). Well, the first delegation would probably convey the idea that earthly firms are places where one confines vicious and cunning people who are made to play extremely sophisticated games according to rules designed in order to prevent them to do much harm to themselves and to others. Only casual mention would be made -if at all- to conventional labels by which the outcomes are denominated (being them "steel", "shoes", "computers" ...), while lengthy accounts would be devoted to the details of the admissible rules and the mathematical equipment humans utilize in order to figure out how to behave.

The second delegation is likely to return with a strikingly different story. It would probably begin with a rather long description of the impressive variety of "things" that each day come out of earthly firms -that is, precisely, steel, computers, polypropylene, etc.- and the equally impressive diversity in the processes leading to them. Moreover, Mars visitors would almost certainly remark that no one has the entire plan of what to do in their heads. Most of the members of each organization repeatedly undertake recognizably few operations and still organizations coordinate their tasks in ways generally yielding coherent artifacts at the end of the day. Indeed this second delegation is likely to suggest the analogy of a "firm" with a messy but most often reliable computer program, with little mention of possible conflict of interests among the individual carriers of various "sub-routines."

Notwithstanding its being a caricature, the foregoing story does convey the spirit of an actual major divide cutting across current theorizing about organization, having at the two extremes a pure incentive-governance view vs. a pure problem solving view. Clearly, there are elements of truth in both perspectives (Coriat and Dosi, 1988a). An ambitious research program would be to connect and unit the two world views.

The starting point for such bridge building has important consequences for the sort of birdge that one creates. The starting point embodies a commitment to some assumptions on first order vs. second order effects. Forced to such a choice, we would pick the second weltanschauung as a provisional point of departure (which also happens to be the least explored one). We do need to assume a weak incentive compatibility to begin with (see Dosi and Marengo (1993)) in the loosest sense that there exists some selection pressure which, in turn, generates some connection between performance and rewards. However, having that, one precisely focuses (as a first theoretical approximation) on the diverse problem solving characteristics of different organizations, and only in the second instance one tackles the ways incentive structures shape/co-evolve with problem solving knowledge. 
Putting it in another way, the archetype "incentive view" fully censors any competence issue associated with what organizations do and how well they do it -except for issues of misrepresentations of "intrinsic" individual abilities and adverse selection, or incentive misalignement in effort elicitation, so that , in the extreme, given the "right incentives" (?) anyone can make microprocessors as well as Intel, bioengineering as Genetech, and even the authors of this paper would easily provide an elegant two-pages proof of Fermat Last Theorem.

The second, "problem solving", archetype, on the contrary, censors precisely the incentivealignment issue. In a sense, all agents are "angels" as their motives are concerned. Conversely, it focuses on the problem solving efficacy of what they do, especially in so far as what they do does not stem from any differential "ontological" ability (if you want you might take the view that the mass of the distribution is on idiots everywhere, or on under-utilized geniuses everywhere) but rather from the social division of tasks and their combinatorics.

So, in the first approximation of this latter view, the basic units of analysis are elementary physical acts -such as moving a piece of iron from one place to another- and elementary cognitive acts -such as applying inference rules. Problem solving can be straightforwardly understood as combinations of elementary acts, within a procedure, leading to a feasible outcome (e.g. an engine, a chemical compound, etc.). ${ }^{2}$

One can also describe it the other way round. Given all the problem solving procedures leading to a given "outcome" (e.g. an engine, etc. and, for that matter, a theorem, a statement about the purported structure of the observed world) -which might well be an infinite set- one may decompose them in subsequences of elementary acts of varying length that may be eventually performed according to various execution architectures (e.g. sequential, parallel, hierarchical...) At this level of analysis, an organization embodies problem solving in at least three senses. First, it displays the operational competencies associated with its actual problem solving procedures (much in accordance with the routines discussed in Nelson and Winter (1982); see also Cohen et al. (1996)). Second, the organizational structure -both the formal and informal ones- determines the distribution of informational inputs of the processing tasks and of the "allowable acts" (i.e. "who can do what to whom") and as such it determines all the decompositions of problem solving procedures that are, so to speak, "legal". Third, it shapes the search heuristics for yet-unsolved problems -e.g. a new engine, a new chemical compound, etc.-, that is, broadly speaking, the heuristics of innovative search.

Note that, although a bit more abstract, this characterization of problem solving knowledge is well in tune with the evidence stemming both from the economics of innovation (for a survey, see

\footnotetext{
2 See Marengo et al (2000) for further discussion of this point.
} 
Dosi (1988)) and capability-based views of organizations (Dosi, Nelson and Winter (2000) among others). Further, it has the advantage of being directly applicable to both the analysis of intraorganizational structures and of the boundaries between organizations and markets. ${ }^{3}$ Indeed, such boundaries may be straightforwardly seen as a particular decomposition of an overall problemsolving task, say the one leading from raw iron oxide to GM engines. Relatedly, one might inquire about the problem solving properties of particular decompositions, ranging from the totally centralised and autarkic one (no decomposition at all), to the analogue of a "pure market" --- one person - one task, with market transactions linking each elementary act. This is a sort of antiCoasian question asking whether, and under what circumstances, markets, i.e. highly decentralized decompositions of knowledge, bear some differential problem solving advantages, if any, when neglecting the more familiar arguments concerning the high powered incentives of markets.

An analysis along these lines is presented in Marengo et al. (2000), where the underlying formal instruments are spelled out with greater detail. Together one finds there a few comparative exercises across different cognitive representations by different organizational structures of the same "true" environments, highlighting their distinctive performance properties. Here, we intend to move some step forward in the direction of incorporating cognitive and political conflict into the analysis, together with (admittedly rudimentary) issues of inventive-compatibility and effort elicitation. Or, putting it again in a sort of caricature, we begin to explore what happens in our stylized organizational models whenever curious, but not omniscient "angels" (i.e. unlimitedly "benign" and collaborative agents) start a) clashing on diverse "visions of the world"; b) facing some hierarchical filtering and/or veto powers on their adaptive search; or, worse, c) they stop being "angels" but react to incentives and orders.

The following section presents the basics of our model. We then engage in some basic analysis of the effect of alternative problem decompositions and reward structures of the adaptive process. Global reward structures, while manifestly the desired payoff scheme for a population of omniscient and compliant actors are not so clearly desirable in the face of not so omniscient, though still compliant problem solvers. Thus, we find that there is an important interaction between problems of knowledge and problems of incentives. The "wrong", excessively local reward structure, may help compensate for the problem of incorrect cognitive representations.

Narrow, group-wide incentives create conflict of interests among problem-solving teams. These conflicts, in turn, help prevent organizations from locking into modest local peaks. However, these conflicts have the dysfunctional property that they may be unending and result in the

\footnotetext{
${ }^{3}$ In the latter respect, it bears at least the same generality as the representation in terms of elementary transactions adovcated by Williamson.
} 
continual perturbation of the problem space of one sub-unit, or actor, by another. We find that there is a further complement, a complement neither from the world of incentives or cognitively limited automatons but from the world of politics --- that is, power. Power may be horizontal, in that one sub-unit is privileged above others, or, as more traditionally viewed, vertical. In either form, the presence of a asymmetrical power distribution within the organization helps to stem the potentially endless cycling of self-perturbing changes that result from incorrect decompositions of the actors' problem environment. Concretely, we explore how the ability of one sub-unit or a hierarchically superior to veto proposed policy changes may enhance the performance of the system.

Finally, we explore the more direct costs of problem decompositions and change efforts. Decompositions have costs not only as a result of the fact that they may incorrectly capture the true structure of the problem space, but more refined structures in and of themselves are difficult to sustain. Similarly, change efforts not only run the risk of yield an inferior policy choice, they are also effortful. Maintaining an established routine surely requires less effort than initiating a new action pattern. We revisit the interaction among cognitions (in the form of problem decompositions) and reward structures while incorporating these features of the cost of more refined problem decompositions and the direct costs of organizational change. Correct decompositions may suffer not only because of the cost of sustaining refined problem decomposition but also because they may induce actors to engage in excessive search.

A subtle but crucial issue we will touch upon, concerns the nature and role of power, if at all, in the interpretation of what (economic) organizations are and of the way they function.

Not surprinsingly, the "power variable" doe not have an independent status in most standard agency theories of the firm. Somewhat more surprinsingly, it does not either in transaction cost theories of organization (cf. Williamson (19 )). Conversely, here we try to implement a notion of power well in tune with a long stream of thought certainly covering Max Weber and Herbert Simon (but dating back at least to St. Augustin), whereby the ruler 1) defines the choice set of the ruled, 2) may prescribe the choice algorithm or the action itself to the ruled, 3) may well shape the very structure of inner goals of the ruled agents to its own objectives.

In fact, Kreps' summary (Kreps (1996))of Simon's seminal labour relation model (cf. Simon (1951)) is a concise and crisp statement of the former two properties:

"... The contract is incomplete in that the employer and the employee are presumed unable to write an enforceable contingent contract specifying what the employee will do as a contingent function of the state of the world. Governance enters in the presumption that the employer will determine ex-post what task the employee will undertake" (Kreps, 1995, p.567). 
While this definition captures a few fundamental ingredients of power relations which are blackboxed under the incomplete contracts heading, it does also hint at some difficult issues which shall begin to address below, that is the consequences of the inability of the ruler/ principal/ manager to correctly specify the prescribed action. Indeed, the foregoing archetype look closely alike the taylorist/fordist dream discussed in Coriat and Dosi (1998) and, from a different angle, in Aoki (2001). What happens to organizational behaviours and performances if organizational adaptation and competitiveness requires active problem-solving efforts by the "ruled ones"? And, more generally, how do patterns of task assignments, with their inherent power dimension, couple with learning patterns?

\section{Model Structure}

We explore complex, though structured problem surfaces. That is, following on recent work on rugged landscapes (Kauffman, 1993; Levinthal, 1997), we highlight the role of interactions among policy choices. However, in contrast to this line of modeling, we follow Simon's argument regarding the prominence and importance of decomposable systems (Simon, 1969) and recent modeling work by Page (1996) and Marengo (2000). Interactions are present among policy choices, however these interactions are not disbursed randomly among the set of possible combinations. Rather, they are tightly clustered within decompositions of the broader system. However, in keeping with the tradition of bounded rationality (Simon, 1955) and in contrast to many recent examinations of modular systems (cf., Baldwin and Clark, 2000), we do not presume that this interaction structure is known to the actors. The partitioning of the decision problem may or may not correspond to the true decomposition structure. A critical element of our analysis is how the appropriate reward structure depends on the degree to which the task partitioning corresponds to the actual structure. In particular, we find that local reward structures may compensate for overly narrow task partitions.

Landscapes are generated according to the following procedure. Let $\mathrm{N}$ be the number of bits or policy choices ( $\mathrm{N}=20$ in the simulations below). The decomposition defines the nature of epistatic interactions among these policy choices. For simplicity, we assume that policy choices within a partition are fully interactive, all elements of the partition interact with all other elements within the partition, while policy choices are assumed to have no interdependence with choices outside the partition. In this sense, we are assuming the problem corresponds to a fully 
decomposable system. We use the following decomposition scheme as a baseline characterization of the problem space:

$\mathrm{D}=\{\{1,2,3,4,5\},\{6,7,8,9,10\},\{11,12,13,14,15\},\{16,17,18,19,20\}\}$

This structure implies that the 20 dimensions of the landscape can be decomposed (cf. Marengo 2000) into 4 separate blocks of 5 dimensions each, and each of these blocks can be optimised independently of the others. However, given that actors may adopt an incorrect partition, the false representation may induce interaction effects across the perceived decompositions

Individual fitness contributions for each bit, or policy choice, are generated by assigning a random number from a uniform distribution between 0 and 1 . Each bit has $2^{\mathrm{k}}$ individual fitness values, where $\mathrm{k}$ is the size of the block to which it belongs. For instance, suppose that $\mathrm{N}=6$ and $\mathrm{D}=\{\{1,2,3\},\{4,5,6\}\}$ is the true decomposition scheme. A random assignment of individual fitness values $f_{i}(i=1,2 \ldots 6)$ takes on the following form:

\section{Table 1 - Example of individual fitness values}

\begin{tabular}{llllllll}
\hline Bit & Block & $\mathbf{f}_{\mathbf{1}}$ & $\mathbf{f}_{\mathbf{2}}$ & $\mathbf{f}_{\mathbf{3}}$ & $\mathbf{f}_{\mathbf{4}}$ & $\mathbf{f}_{\mathbf{5}}$ & $\mathbf{f}_{\mathbf{6}}$ \\
\hline $\mathbf{0}$ & $\mathbf{0 0}$ & 0.29 & 0.73 & 0.64 & 0.99 & 0.83 & 0.35 \\
$\mathbf{0}$ & $\mathbf{0 1}$ & 0.67 & 0.68 & 0.28 & 0.24 & 0.75 & 0.03 \\
$\mathbf{0}$ & $\mathbf{1 0}$ & 0.74 & 0.33 & 0.18 & 0.34 & 0.55 & 0.69 \\
$\mathbf{0}$ & $\mathbf{1 1}$ & 0.63 & 0.63 & 0.57 & 0.33 & 0.54 & 0.46 \\
$\mathbf{1}$ & $\mathbf{0 0}$ & 0.41 & 0.19 & 0.47 & 0.76 & 0.58 & 0.48 \\
$\mathbf{1}$ & $\mathbf{0 1}$ & 0.25 & 0.58 & 0.67 & 0.74 & 0.89 & 0.58 \\
$\mathbf{1}$ & $\mathbf{1 0}$ & 0.55 & 0.64 & 0.44 & 0.56 & 0.34 & 0.73 \\
$\mathbf{1}$ & $\mathbf{1 1}$ & 0.85 & 0.67 & 0.39 & 0.08 & 0.55 & 0.47 \\
\hline
\end{tabular}

The table indicates that when the first bit is set to 0 , its fitness value is 0.29 if the second and third bit are both set to 0 . In contrast, the fitness value of a 0 in the first bit is 0.67 if the second is set to 0 and the third to 1 , while it take on a value of 0.63 if they are both set to 1 . Analogously, if the $5^{\text {th }}$ bit is set to 1 , its fitness value is 0.58 if the $4^{\text {th }}$ and $6^{\text {th }}$ bits are both equal to 0 , while it takes on a value of 0.89 if the $4^{\text {th }}$ equals 0 and the $6^{\text {th }}$ equals 1 , and so on.

In addition to considering the fitness contribution of a individual policy choice, possibly contingent on other choices, we wish to consider the value of broader aggregations of policy strings. Here we follow the lead of Kauffman (1993) in assigning fitness value to broader systems by merely averaging the fitness contributions of the individual elements; however, in contrast to this 
earlier tradition, we are sensitive to the possibility of different levels of aggregation. Performance, and in turn rewards, may be based on the fitness value of the entire policy string, or some partition of this broader string. In particular, a special case of more refined partitions is the set of partitions consisting of singletons, or individual policy choices.

Using the illustrative fitness table provided in Table 1 above, we can demonstrate this notion of evaluating performance at different levels of aggregation. Consider the string 101110 . We can define three notions of fitness:

1) individual fitness, which are respectively:

$\mathrm{f}_{1}=0.25, \mathrm{f}_{2}=0.63, \mathrm{f}_{3}=0.44, \mathrm{f}_{4}=0.56, \mathrm{f}_{5}=0.34, \mathrm{f}_{6}=0.46$

2) block fitness, defined for each of the two blocks of the decomposition scheme:

$$
\begin{aligned}
& F_{\{1,2,3\}}=\left(f_{1}+f_{2}+f_{3}\right) / 3=(0.25+0.63+0.44) / 3=0.44 \\
& F_{\{4,5,6\}}=\left(f_{4}+f_{5}+f_{6}\right) / 3=(0.56+0.34+0.46) / 3=0.453
\end{aligned}
$$

3) global fitness (of the entire string):

$$
\mathrm{F}=\left(\mathrm{F}_{\{1,2,3\}}+\mathrm{F}_{\{4,5,6\}}\right) / 2=\left(\mathrm{f}_{1}+\mathrm{f}_{2}+\mathrm{f}_{3}+\mathrm{f}_{4}+\mathrm{f}_{5}+\mathrm{f}_{6}\right) / 6=0.4465
$$

These same three levels, individual, block, and global not only can be the basis for evaluating actual performance at different levels of aggregation, but they can also define the search space by which alternatives are generated and evaluated. The nature of alternative generation is determined by way the choice problem is decomposed. The organisation explores the landscape according to its own decomposition scheme $\Delta$, which may or may not coincide with the "real" decomposition scheme D (i.e. the one which generates the landscape). Different actors, or subunits within the organisation may explore each of the partitions of the decomposition. Consider, for instance, the illustrative 6 bit landscape provided above. Suppose that this landscape is explored by an organisation characterised by the following incorrect decomposition scheme:

$\Delta=\{\{1,2\},\{3,4,5,6\}\}$. Search within a block of the decomposition $\Delta$ (in our example either $\{1,2\}$ or $\{3,4,5,6\})$ involves mutating at least one and at most all the bits of the selected block. ${ }^{4}$ We explore settings in which we restrict the mutuation to be a change in a single bit within the decomposition (corresponding to ideas of local search (Levinthal, 1997), as well as settings, following Marengo (199x), in which one of the full set of possibilities is drawn at random.

Independent of the problem decomposition, the reward structure faced by the actors may be more or less aggregate. For instance, the imposed decomposition of the problem space may be quite narrow; however, each subunit could still be evaluated on the basis of the global fitness of the

\footnotetext{
${ }^{4}$ In our example, if block $\{1,2\}$ is selected, mutation could produce one of the following three strings: $100000,010000,110000)$.
} 
overall system. As a result, in such a setting, action would be 'local', while thinking would be 'global'. The benefit of having a reward structure that maps more or less closely to the task partition depends, in turn, on the degree to which the task partition itself reflects the actual problem decomposition.

Thus, under a global reward structure, a new policy alternative is adopted if it enhances the fitness of the overall system. Depending on the problem decomposition, this policy initiative could consist of a single, one-bit mutation or, under more aggregate problem decompositions, it could consider of more radical changes in the set of policies. Under a block, or team reward structure, proposed alternatives are viewed favorably if they enhance the fitness level of the set of policies corresponding to the overall partition of the problem decomposition. . Note that this reward scheme is equivalent to the global one if the "conjectural" decomposition $\Delta$ and the "real" one D are identical. With individual level rewards, a proposal is viewed favourably if the fitness contributed of the particular policy choice being changed increases.

Let us clarify how the three schemes actually work by referring again to the above example. Suppose that fitness values are those of table 1 , that the real decomposition is $D=\{\{1,2,3\},\{4,5,6\}\}$ and the conjectural one is $\Delta=\{\{1,2\},\{3,4,5,6\}\}$ and the current string is 000000 . Suppose now that the block $\{1,2\}$ is selected and the current string gets randomly mutated into 110000 . Let us examine the rewards computed by the three different schemes in this case:

1) global reward:

000000 has global fitness $0.638,110000$ has global fitness 0.667 . Thus 110000 is retained. 2) team reward:

For block $\{1,2\}$ the configuration 00 has fitness 0.51 (i.e. $(0.29+0.73) / 2)$, while 11 has reward 0.595 (i.e. $(0.55+0.64) / 2)$. Thus 11 is retained.

3) individual reward:

For the first bit choice 0 within string 000000 has fitness 0.29 , while choice 1 within string 110000 has fitness 0.55 . Thus choice 1 is retained;

for the second bit, choice 0 within string 000000 has fitness 0.73 , while choice 1 within string 110000 has fitness 0.64 . Thus choice 0 is retained.

All in all, both global and team reward schemes select string 110000, while the individual scheme selects string 100000. 
Each period of the simulation a single block of the perceived decomposition of the problem structure is chosen at random. A new alternative specification for that block is drawn at random. In some of our analyses, we restrict this random draw to consist of a one-bit mutation from the existing policy choice; in other settings, the random draw is not restricted and, as a result, potentially all elements of the partition could be changed in the proposal. The former setting corresponds to ideas of incremental search. In contrast, the later structure assumes that search is limited by the problem decomposition. The new alternative is evaluated on the basis of the reward structure (global, team, or individual) and adopted if it enhances performance from the perspective of the relevant reward structure.

When we explore the role of power on the process of adaptation, a additional criterion is added to the evaluation of a proposal. Proposals must look attractive from the perspective of the initiating subunit, but must also be attractive (i.e., fitness improving) from the perspective of the 'powerful' subunit. Thus, proposal may be subject to vetoes.

\section{Analysis}

The following simulations consider a problem of size $\mathrm{N}=20$, generated by the following “true" decomposition: $\mathrm{D}=\{\{1,2,3,4,5\},\{6,7,8,9,10\},\{11,12,13,14,15\},\{16,17,18,19,20\}\}$

The following figures present results, respectively, for one typical simulation runs and for averages and standard deviations over 200 different simulations. Each of the 200 replications uses the same structure in terms of the partitioning of the problem, but the seeding of the landscape is independently drawn in terms of fitness values.

\section{Alternative Problem Decompositions and Local Search}

The "conjectural" decomposition on which organisational search is based has a double role, which is typical of any division of labour. On the one hand, it defines a "cognitive" structure under which the problem is considered. This cognitive structure translates into a decomposition of the problem into sub-problems, which are treated as if they were independent and, in turn, constrain the portion of the search space that can undergo examination. The perceived decomposition also influences the potential reward structure. In particular, the team, or block reward is a function of what the organisation conceives of as a block. We are interested in exploring this double role, but in the initial simulations we isolate the reward structure definition role by supposing that search always proceeds by one bit mutation only.

Figure 2 illustrates the average performance over 200 replications for a process of local search (one bit mutations) for a conjectured problem decomposition consisting of the minimum 
partitions of single choice variables, i.e., $\Delta=\{\{1\},\{2\},\{3\}, \ldots .,\{N\}\}$, while Figure 1 illustrates a particular run among this set of 200. Given this task partitioning, the indivdual and team based evaluation scheme result in the same evaluation. We also present results for an organization in which one team (the first) has a veto power to forbid all mutations elsewhere in the organization if they cause a decrease of its own fitness level. We will explain at greater length the rationale of this choice later when discussing various notions of hierarchy.

Insert figure 1

Insert figure 2

Insert figure 3

Consider now that the same kind of landscape is explored by an organisation whose conjectural decomposition are "almost" correct. In particular, suppose that the conjectural decomposition catches most of the epistatic interactions but misses some of them, as in the following conjectural decomposition:

$\Delta=\{\{1,2,3,4\},\{5,6,7,8\},\{9,10,11,12\},\{13,14,15,16\},\{17,18,19,20\}\}$

Insert figure 4 here 
Under the global reward structure, the organisations rapidly reach a local optimum in the fitness landscape. ${ }^{5}$ This fact is not surprising. A global reward structure by design only accepts proposed changes that enhance overall system performance.

More interesting, is the behaviour of less aggregate reward structures. Such a reward structure provides, in some sense, a false signal of the value of a proposed alternative. A subunit may accept an initiative (i.e., a change in one of the policy choices) that enhances its own performance, but may degrade the fitness of the overall organisation. As a result, such an organisation may walk "downhill" on a fitness landscape. Indeed, evidence of such occasional downhill movement is provided in the Figure 1 depiction of an individual run. Despite such aberrant behaviour, such organisations, on average, tend to increase their performance. Indeed, in the long-run, they reach similar levels of performance as organisations with a global reward structure.

The conflict generated by local reward structure has functional, as well as dysfunctional implications. The selfish, parochial perspective induced by a local reward structure may cause the organization to adopt new policy strings that are inferior to its prior actions. However, while in an immediate sense such action is dysfunctional, this behaviour also acts to reduce the likelihood that the organization will remain absorbed on an inferior local peak. Indeed, close examination of Figure 2 reveals that even after a 1000 periods, there is still a slight positive gradient to the performance curve for the team=individual reward structure while performance under the global reward structure reaches its maximum value by period 100 and remain fixed at that level in all remaining periods.

Goal conflict acts as an effective substitute for an accurate partitioning of the problem space. With a perfectly accurate decomposition of the problems space, then there is not conflict between the team, or block, evaluation of a proposed initiative and a global perspective. As a result, with an accurate partitioning of the problem, we see no distinction between the long-run performance under

\footnotetext{
${ }^{5}$ A local optimum consists of a point such that any one-bit mutation of the vector of policy choices decreases fitness. However, the fact that a point constitutes a local optimum does not imply that the simultaneous shift in multiple policies could not lead to improved fitness. In general, fitness landscapes with interaction effects among policy choices have multiple local peaks (Kauffman, 1993).
} 
the team and global reward and both these more aggregate structures perform better than an individual reward structure that fails to capture the actual interactions among policy choices. At other extreme settings, the individual and team rewards are one and the same. This occurs, most directly, when the decompositions consists of individual policy choices. In such a case, by definition there is no distinction between individual and team reward.

The following figures provide the results for both these extreme settings: the first of a minimum decomposition of unit bit and the second of a completely miss-specified composition. We see, as argued, that the individual and team reward yield the same performance and that both these reward structures yield an inferior performance to the global reward structure.

\section{Veto Power}

We model veto power in the following way: one of the blocks (or individual bit) of the decomposition $\Delta$ which characterises the organization can stop any mutation of the other blocks (or bits) which decreases its own fitness. Consider, again, the decomposition:

$\Delta=\{\{1,2,3,4,5\},\{6,7,8,9,10\},\{11,12,13,14,15\},\{16,17,18,19,20\}\}$ and assume that block $\{1,2,3,4,5\}$ is endowed with veto power. Any mutation taking place in, for instance, block $\{11,12,13,14,15\}$ will be retained if and only if it increases the team-fitness of block $\{11,12,13,14,15\}$ and it does not decrease the team-fitness of block $\{1,2,3,4,5\}$. When the second condition is not satisfied the mutation is vetoed. Note that veto power is meaningless when the reward scheme is global.

As seen in Figure 7indicating fitness level over time for a single run of the model and Figure 9 providing the standard deviation over a set of 200 iterations of the model, the introduction of veto power has in general a stabilising effect with respect to variability of the corresponding reward scheme. This analysis was done assuming, as before, that the "real" decomposition is $D=\{\{1,2,3,4,5\},\{6,7,8,9,10\},\{11,12,13,14,15\},\{16,17,18,19,20\}\}$, while the organization searches the landscape according to: $\Delta=\{\{1,2\},\{3,4\},\{5,6\},\{7,8\},\{9,10\},\{11,12\},\{13,14\},\{15,16\},\{17,18\},\{19,20\}\}$. Thus, the perceived partitioning of the problem space is more fine-grained than the actual partition. 
Insert Figure 8 here

Insert Figure 9 here

Team and individual reward schemes with veto power are effective in stabilising good solutions when attained. These results appear to be linked to the following factors: 1) the subjective decomposition on which organizational search is based must be finer than the "real" decomposition which originates the landscape and 2) the latter must not be too complex. In fact the result is stronger if we consider a "real world" generated by the following simpler decomposition structure: $\mathrm{D}==\{\{1,2\},\{3,4\},\{5,6\},\{7,8\},\{9,10\},\{11,12\},\{13,14\},\{15,16\},\{17,18\},\{19,20\}\}$

and the organizational decomposition is the finest possible: $\Delta=\{\{1\},\{2\},\{3\}, \ldots .,\{18\},\{19\},\{20\}\}$. In this case, we see that team rewards, in conjunction with veto power, can actually lead to a superior performance than a global reward structure.

In the face of an incorrect decomposition, the conflict that stems from team rewards induces more search than that which results from a global reward structure. This greater search effort may identify a superior solution than that identified under global search; however, a team based reward structure is ineffective in "holding on" to the strong policies that are identified. Under a team reward structure, the organization tends to cycle among possible solutions. One unit initiates changes that appear attractive from its vantage point which, in turn, disrupts the performance of another unit and provides an incentive for that unit to initiate change which in turn feed-backs to the original sub-unit that initiated change, and so on. The presence of a veto breaks such cycling. Only a subset of proposed changes are allowed to become policy; the subset that will not disrupt the performance of the powerful sub-unit.

Insert Figure 10 here 


\section{Decompositions, span of control and elicitation costs}

In this section we extend our model in order to introduce costs which the organization has to bear in order to induce its members to act in a given fashion. What we are trying to model is not a principal-agent relationship stricto sensu, because we do not assume any given relationship between effort and outcome. Rather, we focus instead on control. The principal, the residual claimant of the organizational total payoff, wants to induce the agents to keep performing some given action or to switch to a different one. But when actions are linked by interdependencies, the control function itself cannot be entirely decomposed into independent control problems. Thus, the cognitive dimension (given by the decomposition) and the control dimension interact in some non-trivial way.

In order to explore these issues, we elaborate the model structure by introducing a costly control function and analyse the relationship between conflict arising from "cognitive" factors, that is from incorrect decomposition of the problem, and conflict arising from control and incentives. In particular, we assume the costs of action elicitation grow with the span of control, i.e. the size of elements of the decomposition, and are higher when the principal wants to elicit a change in the agent's action than when he wants to elicit the same behaviour. This assumption is based on two independent premises. First, we assume that agents are naturally adverse to change. Second, we assume that the observation and elicitation of "business as usual" can be routinised and take advantage of standard procedures and devices.

More specifically, in the following analysis we assume that elicitation costs have two components:

1) the "business as usual component": $\mathrm{C}_{1}=\mathrm{c} \times \operatorname{span}^{2}$

2) the "new action component": $C_{2}=c \times n \_$mutation $^{2}$

where span is the size of the blocks of the decomposition and n_mutation is number of bits which have been mutated. Finally, $\mathbf{c}$ is a constant coefficient. Total elicitation costs, $\mathrm{C}$, simply equals $\mathrm{C}_{1}$ $+\mathrm{C}_{2}$.

The principal obtains the profit which is given by the total fitness of the organization after payment of the total effort elicitation costs: $\Pi=\mathrm{F}-\mathrm{C}$. We assume that the principal is profit- (rather than fitness-) seeking. That is, the principal incorporate the effect of the cost of effort in determining whether a policy is enhancing or diminishing performance. 
Simulation results:

Again, we consider a landscape with $\mathrm{N}=20$ and generated by the following decomposition: $D=\{\{1,2,3,4,5\},\{6,7,8,9,10\},\{11,12,13,14,15\},\{16,17,18,19,20\}\}$. We explore four classes of perceived decompositions:

1) Right, have the correct decomposition

2) Almost right, use the size four decomposition:

$$
\Delta=\{\{1,2,3,4\},\{5,6,7,8\},\{9,10,11,12\},\{13,14,15,16\},\{17,18,19,20\}\}
$$

3) Wrong: have a decomposition of correct size but biased:

$$
\Delta=\{\{1,2,3,19,20\},\{4,5,6,7,8\},\{9,10,11,12,1314,15\},\{14,15,16,17,18\}\}
$$

4) Minimal: have the finest decomposition:

$$
\Delta=\{\{1\},\{2\},\{3\}, \ldots \ldots,\{18\},\{19\},\{20\}\}
$$

The following figures report respectively fitness and profit for a typical simulation with a cost coefficient $\mathrm{c}=0.01$.

Insert figure here 12

Insert figure here 13

Agents with the right decomposition both in terms of profit (and this was expected because of costs proportional to the span of control) but also in terms of fitness. The former result is due to the fact that fitness improving changes may be too costly (because of the $\mathrm{C}_{2}$ component) and reduce profits. Smaller than right decompositions have higher performance both in profit and in fitness and will tend to prevail in the population. An additional disadvantage of using large decomposition is that if they are wrong the performance becomes extremely poor. 
The virtues of a false decomposition in conjunction with the challenge of motivating and monitoring action are compounded in the context of a more complex problem environment. Consider the environment generated by the following decomposition of size 10:

$\mathrm{D}=\{\{1,2,3,4,5,6,7,8,9,10\},\{11,12,13,14,15,16,17,18,19,20\}\}$. In this setting, we model four types of agents characterised by decompositions of different sizes, called respectively:

1) $\mathbf{K}=\mathbf{1}$, with decomposition:

$$
\Delta=\{\{1\},\{2\},\{3\}, \ldots \ldots,\{18\},\{19\},\{20\}\}
$$

2) $\mathbf{K}=\mathbf{2}$, with decomposition:

$$
\Delta=\{\{1,2\},\{3,4\}, \ldots \ldots,\{17,18\},\{19,20\}\}
$$

3) $\mathbf{K}=\mathbf{4}$, with decomposition:

$$
\Delta=\{\{1,2,3,4\},\{5,6,7,8\},\{9,10,11,12\},\{13,14,15,16\},\{17,18,19,20\}\}
$$

4) $\mathbf{K}=\mathbf{5}$, with decomposition:

$$
\Delta=\{\{1,2,3,4,5\},\{6,7,8,9,10\},\{11,12,13,14,15\},\{16,17,18,19,20\}\}
$$

To highlight the effect the interaction between problem decompositions and problems of control, we contrast the behaviour of a system with agents that require control with that of a system with "angels" --- a set of actors that do not require such control measures.

When these cooperating individuals (i.e., angels) are also perfectly knowledgeable, then we get the expected result that cooperative actors perform better than those agents that require control measures. However, if actors have the wrong cognitive representation of the problem space, that is they enact an incorrect partition, then the non-cooperative agents may actually generate a higher level of performance. The following figure compares the fitness achieved by an organization in which the cooperating individuals have a high dimensional representation of the environment that corresponds in partition width to the true problem representation but the elements are not correctly assigned with agents that require control who have a "simplistic" view of the problem space as consisting of two element partitions.

Insert figure 14 


\section{Conclusions}

- TO BE COMPLETED -

\section{References:}

Dosi, G. (1988), Sources, Procedures and Microeconomic Effects of Innovation, Journal of Economic Literature, vol. 26, pp. 1120-1171.

Dosi G., Marengo L., Toward a Theory of Organizational Competencies, in R.W. England (ed.), Evolutionary Concepts in Contemporary Economics, Ann Arbor, Michigan University Press, 1995, pp. 157-178.

Dosi, G. R. Nelson and S. Winter (eds.) (2000), The Nature and Dynamics of Organizational Capabilities, Oxford, Oxford University Press.

Gavetti G. and D. Levinthal (2000),

Grossman S. and O. Hart (1986), The Costs and Benefits of Ownership: A Theory of Vertical and Lateral Integration, Journal of Political Economy, vol. 94, pp. 691

Kauffman, S.A. (1993), The Origins of Order, Oxford, Oxford University Press.

Kreps, M.D. (1996), Markets and Hierachies and (Mathematical) Economic Theory, Industrial and Corporate Change, vol. 5, pp. 561-595.

Levinthal, D. (1997), Adaptation on rugged landscapes, Management Science, vol. 43, pp. 934-950.

Marengo, L. (2000), Decentralisation and market mechanisms in collective problem-solving, mimeo, University of Trento.

Marengo, L., G. Dosi, P. Legrenzi and C. Pasquali (2000), The Structure of Problem-solving Knowledge and the Structure of Organizations, Industrial and Corporate Change, vol. 9 , pp.757-788.

Milgrom, P. and J. Roberts (1995), Complementarities and Fit: Strategy, Structure and Organizational Change in Manufacturing, Journal of Accounting and Economics, vol. 19, pp. 179-208.

Nelson, R.R. and S.G. Winter (1982), An Evolutionary Theory of Economic Change, Cambridge, Mass., Harvard University Press.

Page, S.E. (1996), Two measures of difficulty, Economic Theory, vol. 8, pp.321-346.

Radner, R. (1986), The Internal Economy of Large Firms, Economic Journal, vol. 96 (supplement), pp. 1-22. 
Simon, H. (1951), A Formal Model of the Employment Relationship, Econometrica, vol. 19, pp. 293-305.

Simon, H. (1969), The Sciences of the Artificial, Cambridge Mass., MIT Press.

Winter, S.G. (1988), On Coase, Competence and the Corporation, Journal of Law, Economics and Organization, vol. 4, no. 1 . 
Figure 1: One Bit Mutation on Minimal Decomposition (Single run)

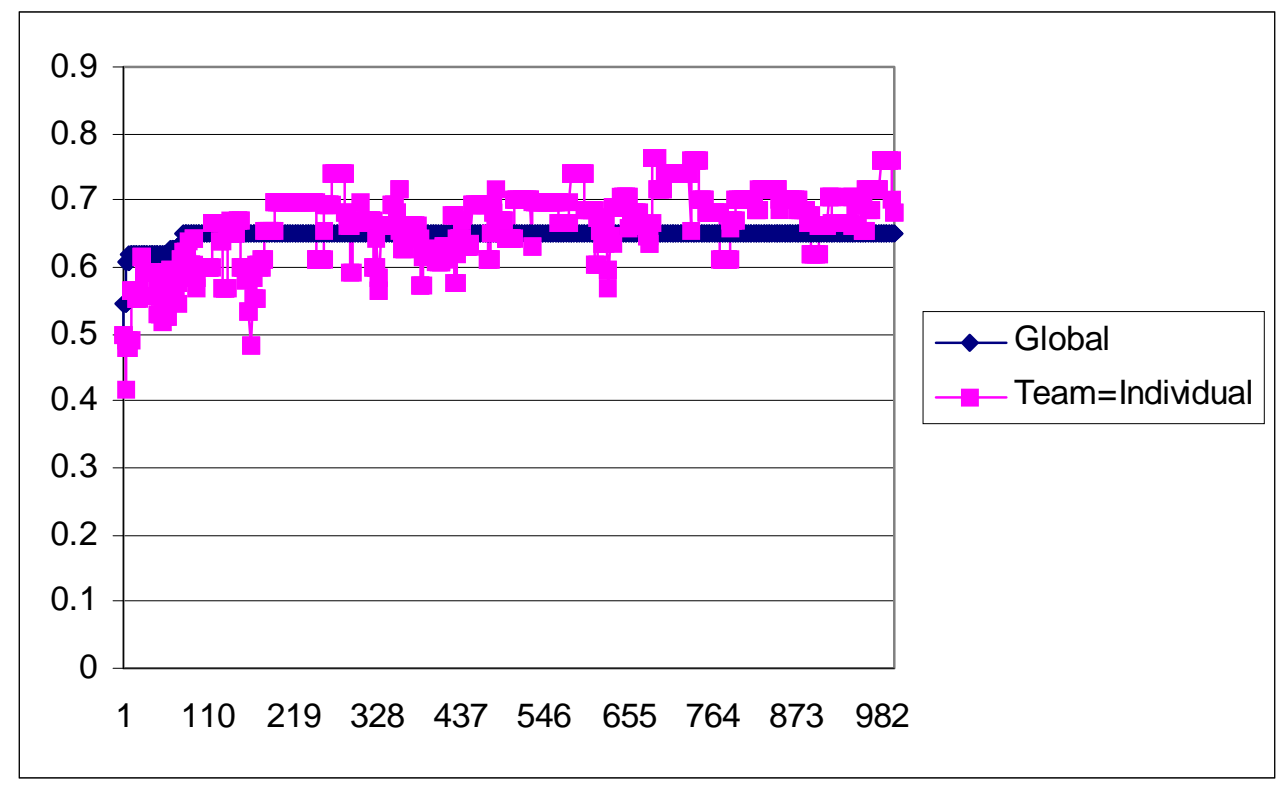

Figure 2: One Bit Mutation on Minimal Decomposition (Average over 200 repetitions)

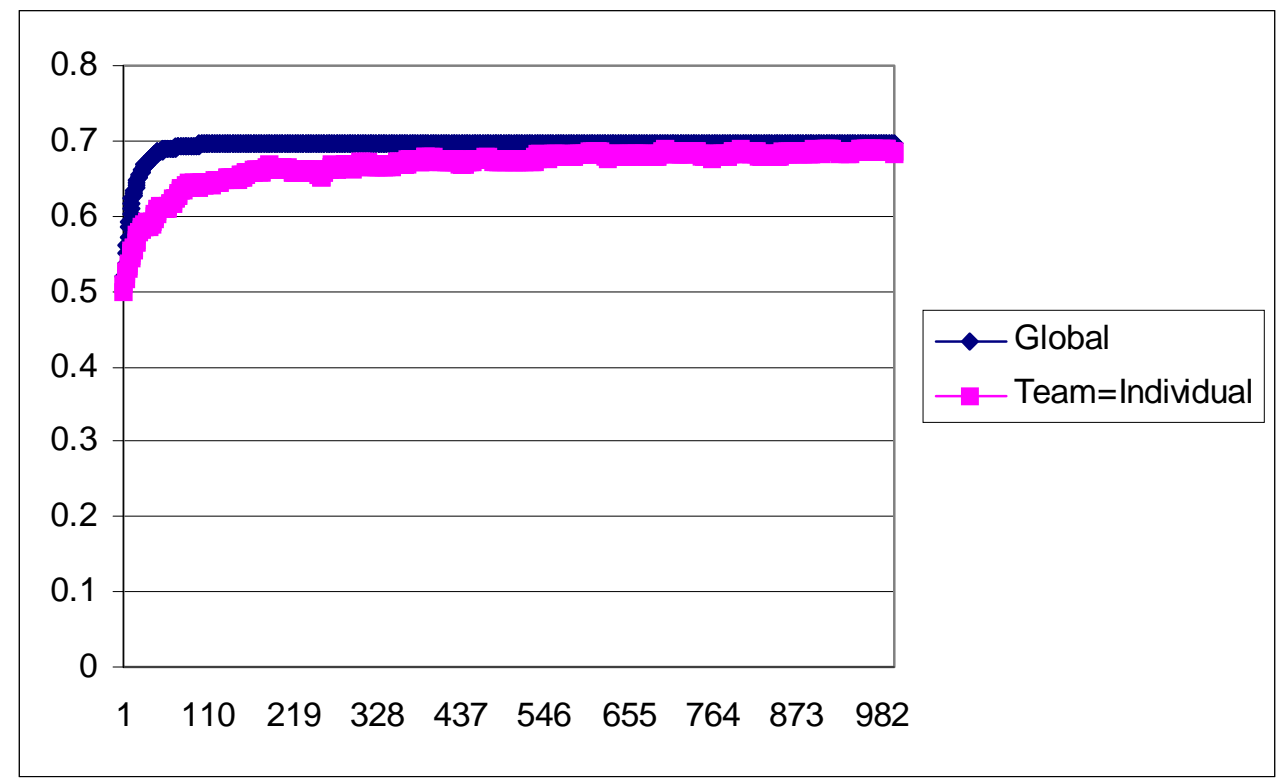


Figure 3: One Bit Mutation on Minimal Decomposition

(Standard deviation over 200 repetitions)

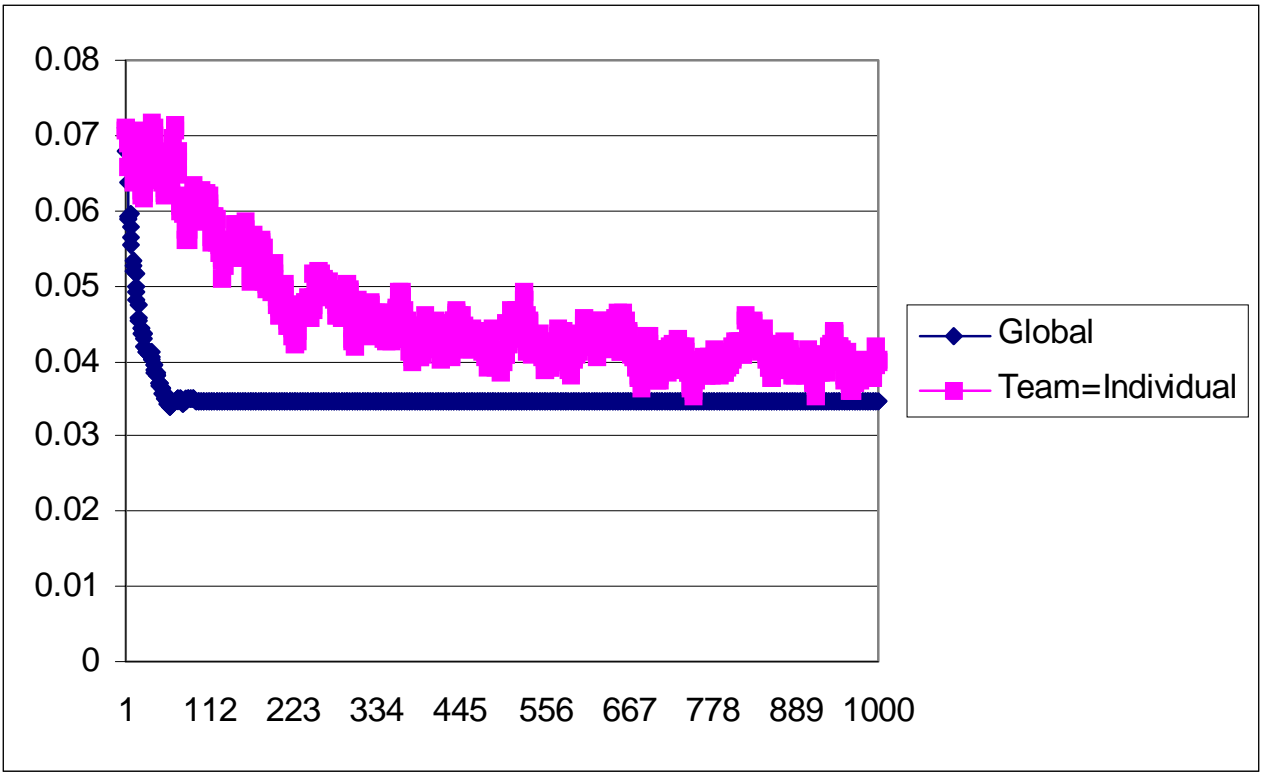

Figure 4: One Bit Mutation on "Almost right" Decomposition (Single run)

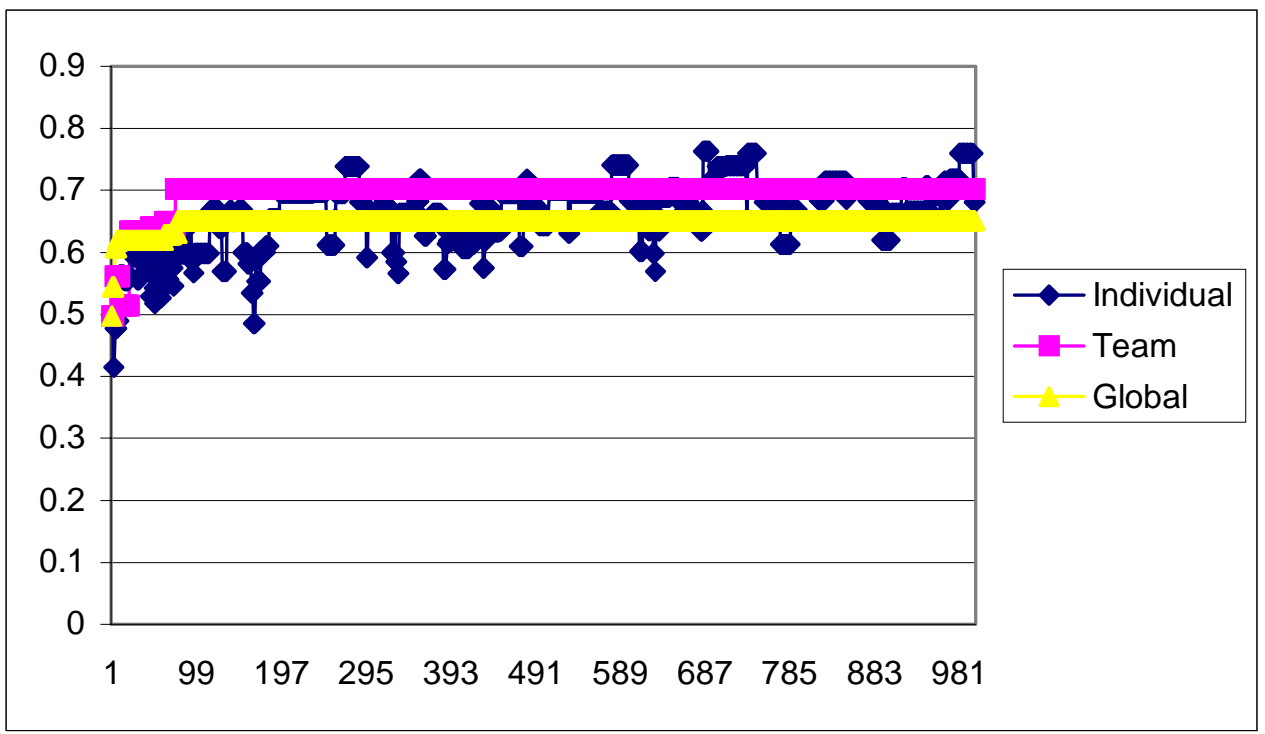


Figure 5: One Bit Mutation on "Almost right" Decomposition (Average fitness over 200 repetitions)

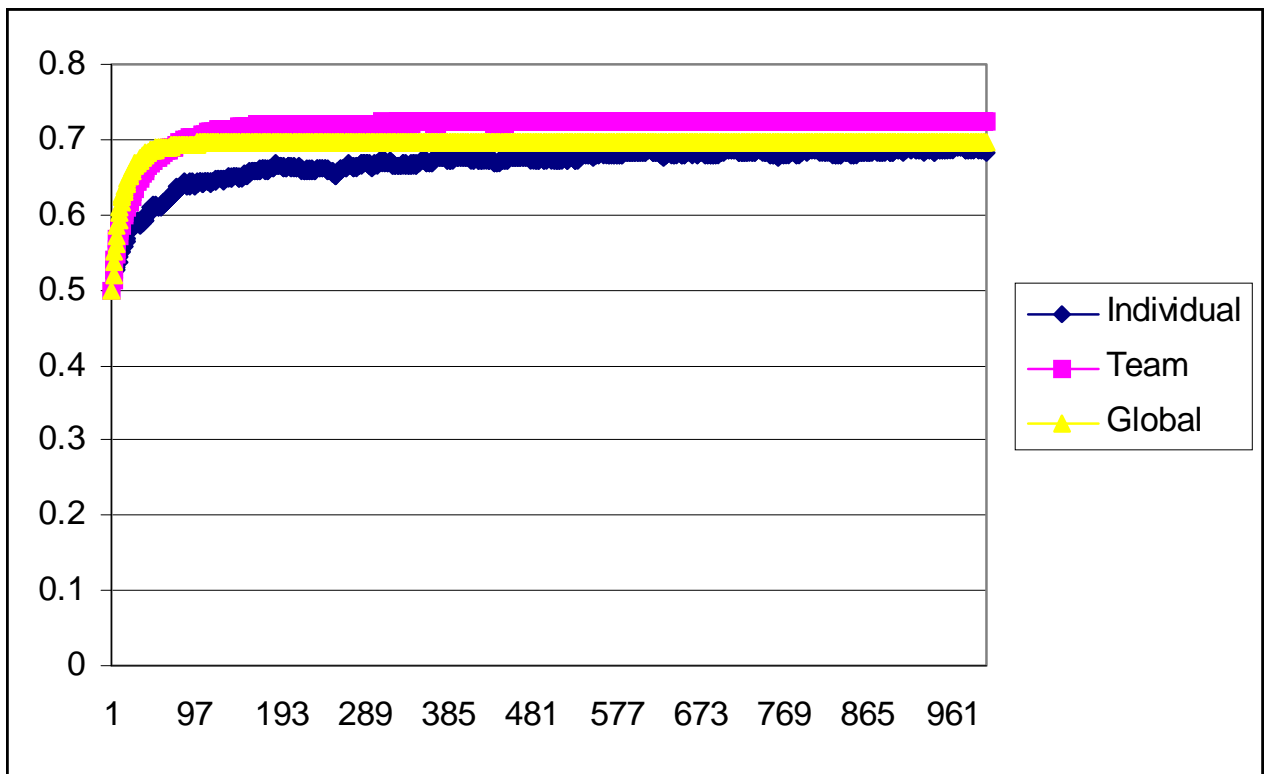

Figure 6: One Bit Mutation on "Almost right" Decomposition (Standard deviation across 200 repetitions)

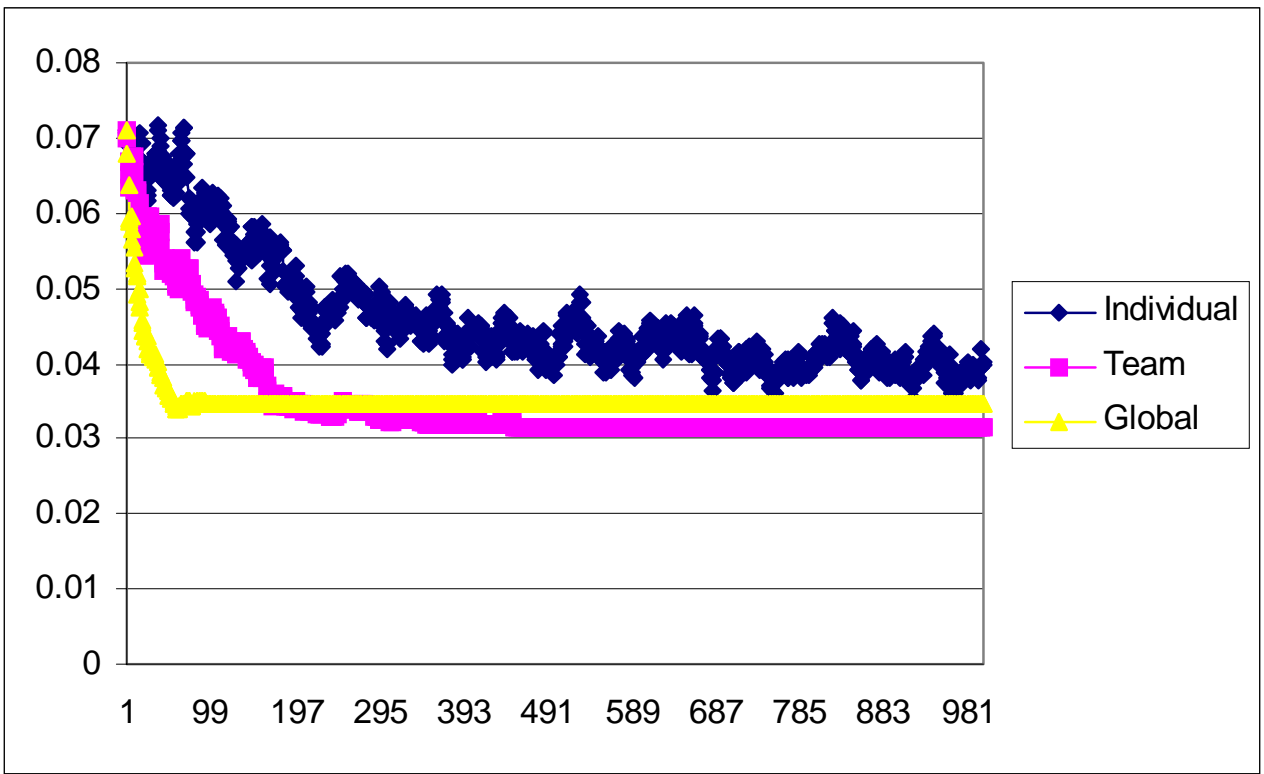


Figure 7: Veto Power with Partitions of Two

(Single run)

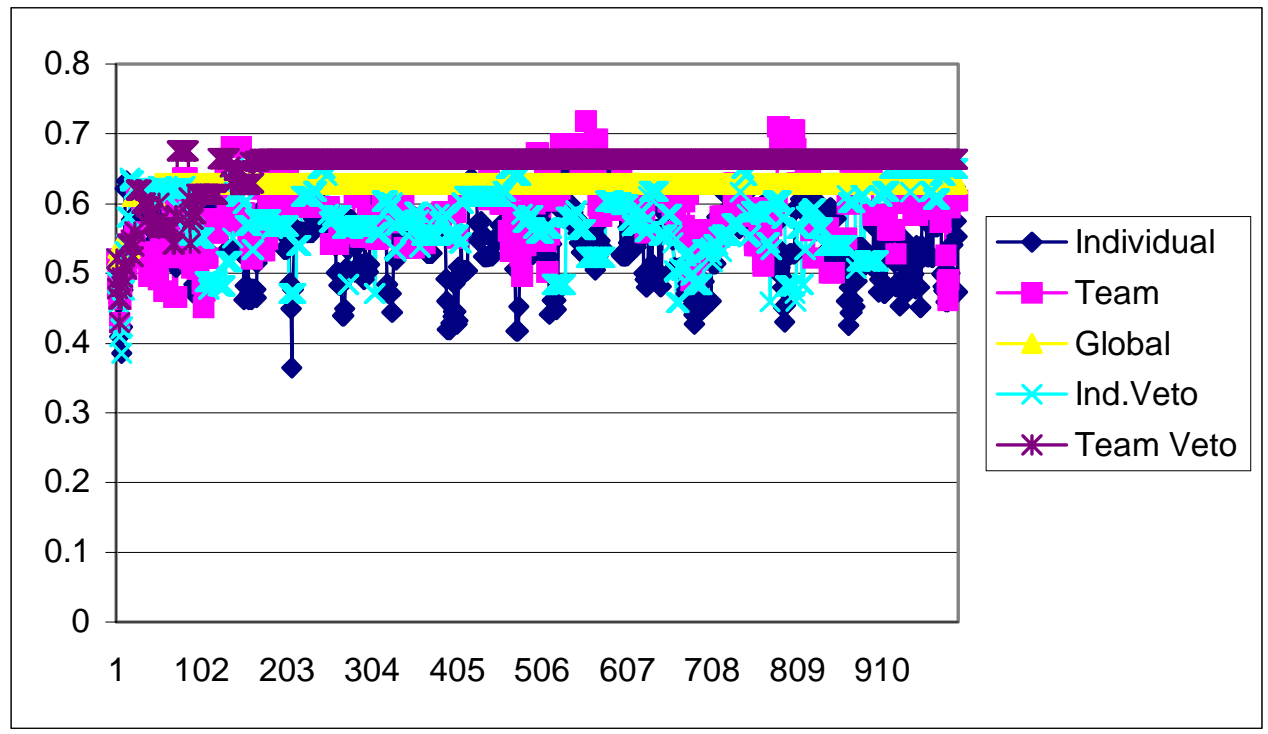

Figure 8: Veto Power with Partitions of Two

(Average fitness across 200 repetitions)

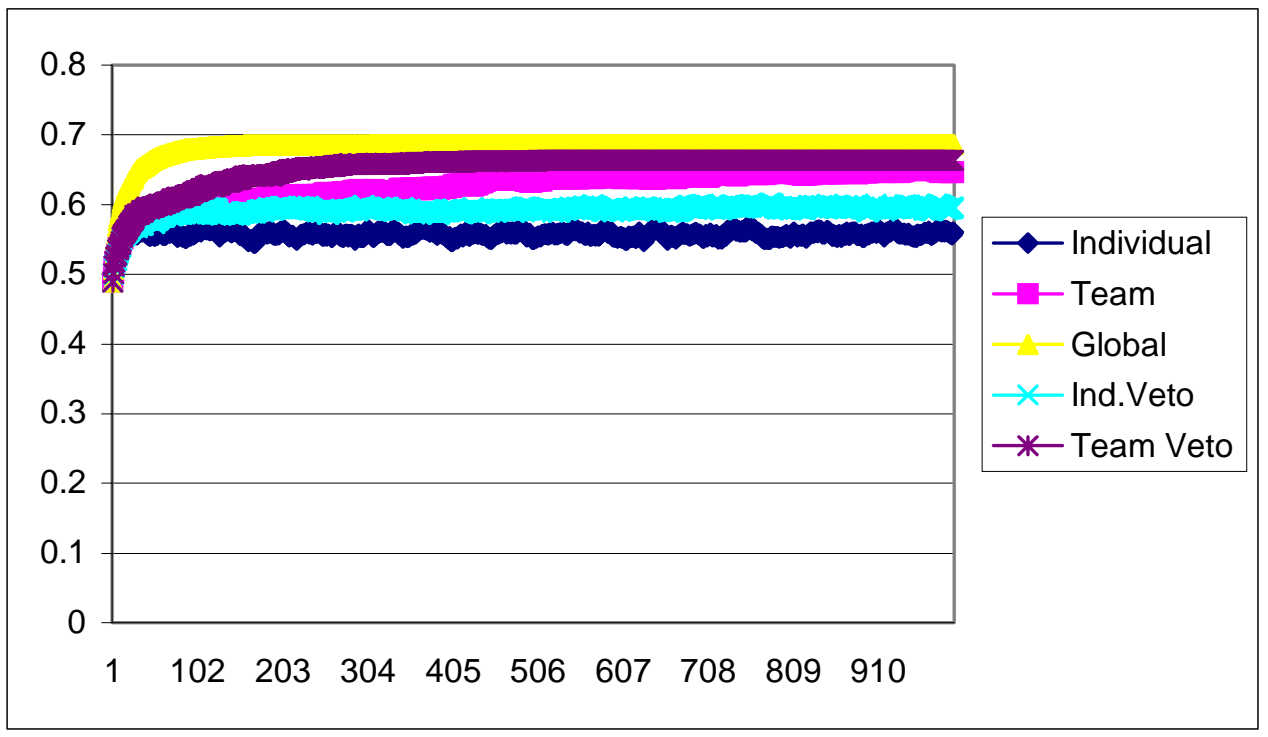


Figure 9: Veto Power with Partitions of Two

(Standard deviation across 200 repetitions)

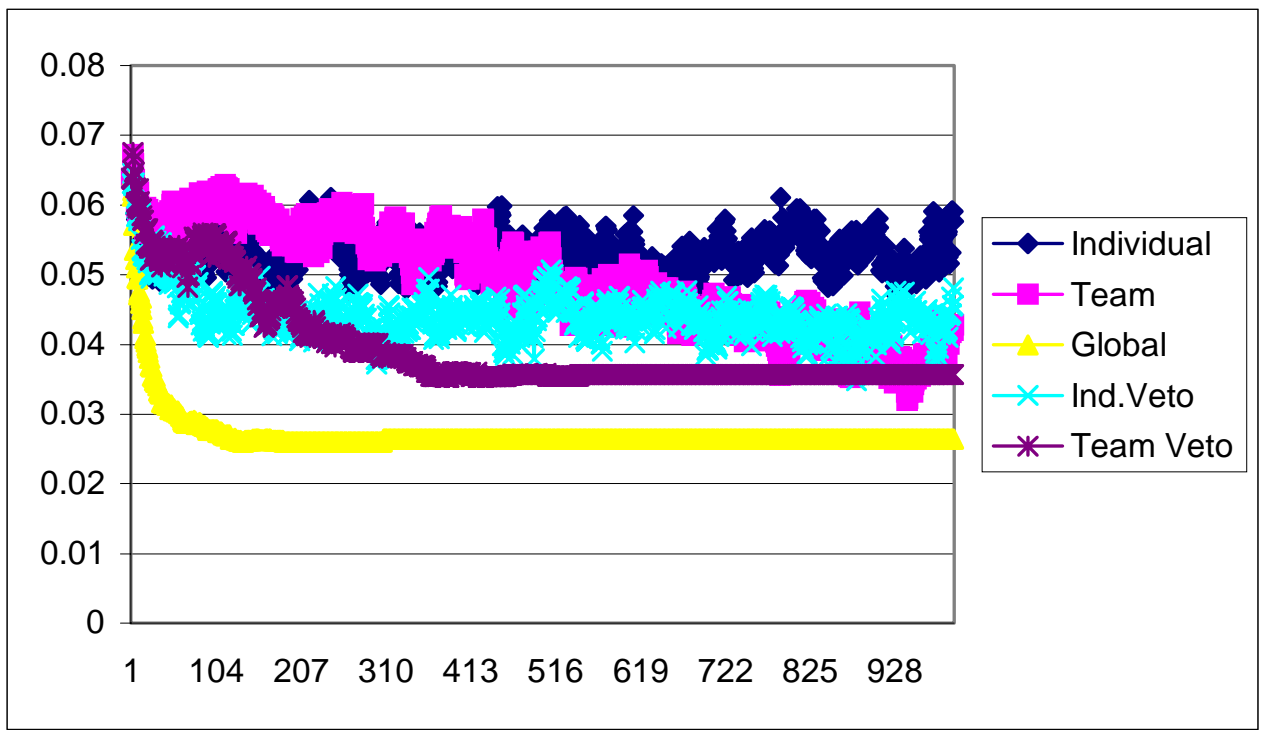

Figure 10: Veto Power with Perceived Partitions of One and Real Partitions of Two (Average fitness across 200 repetitions)

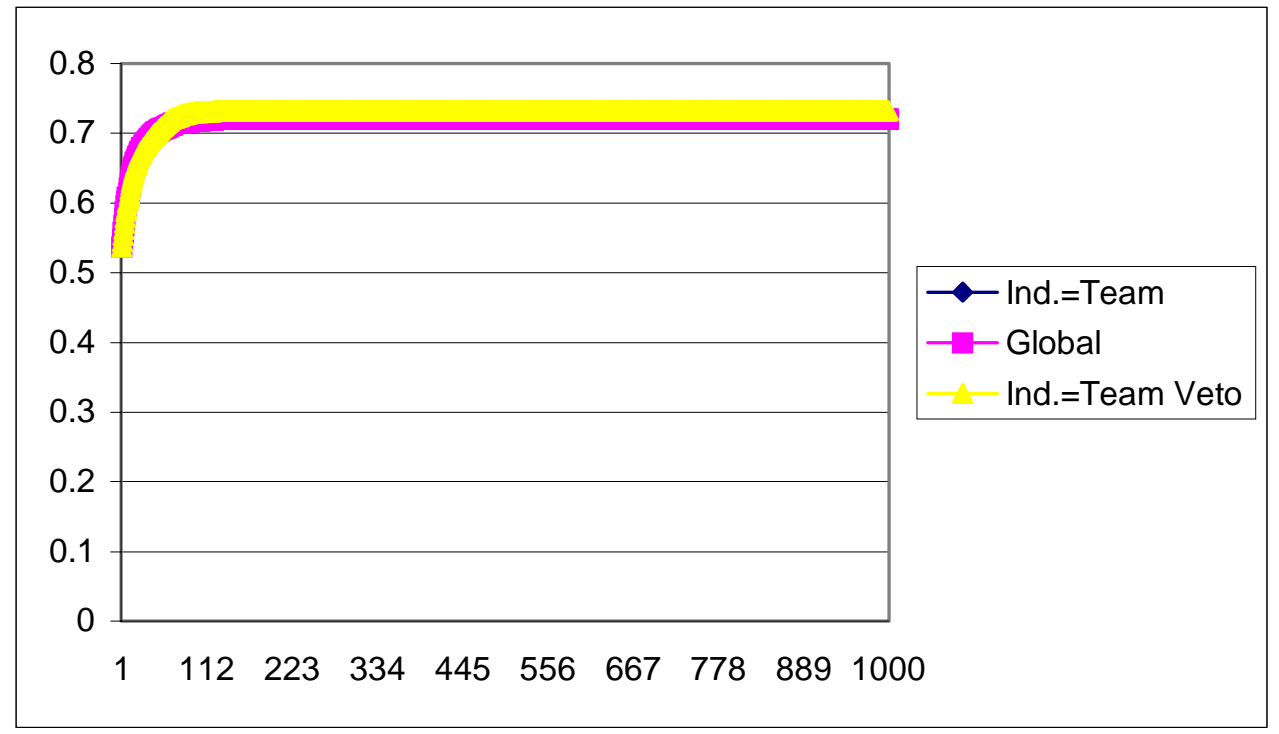


Figure 11: Veto Power with Perceived Partitions of One and Actual Partitions of Two (Standard deviation across 200 repetitions)

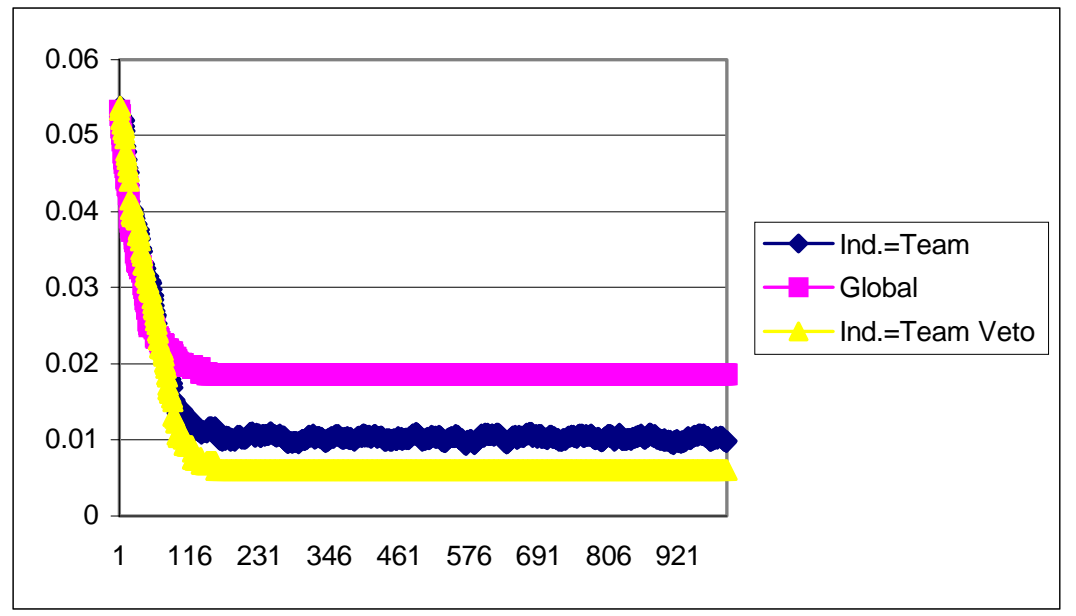

Figure 12: Agency Costs

(Average fitness level over 200 repetitions)

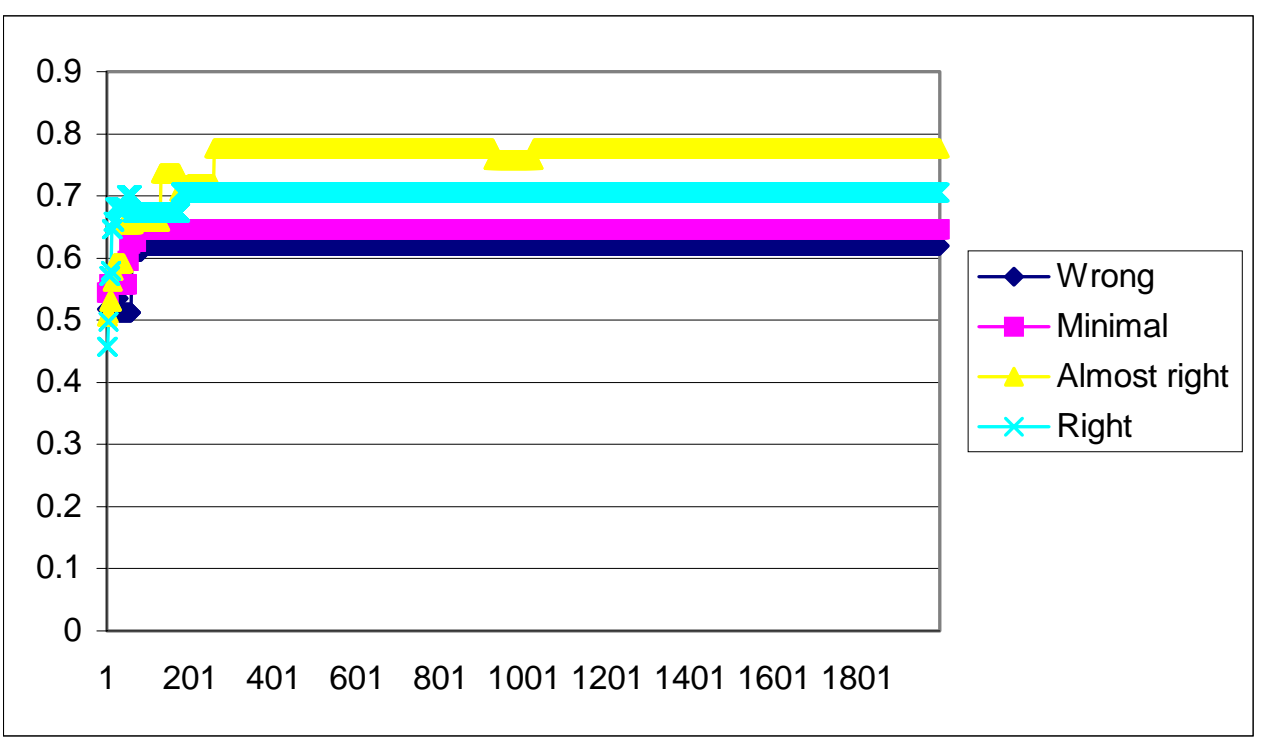

Figure 13 : Agency Costs

(Average profit value over 200 repetitions) 


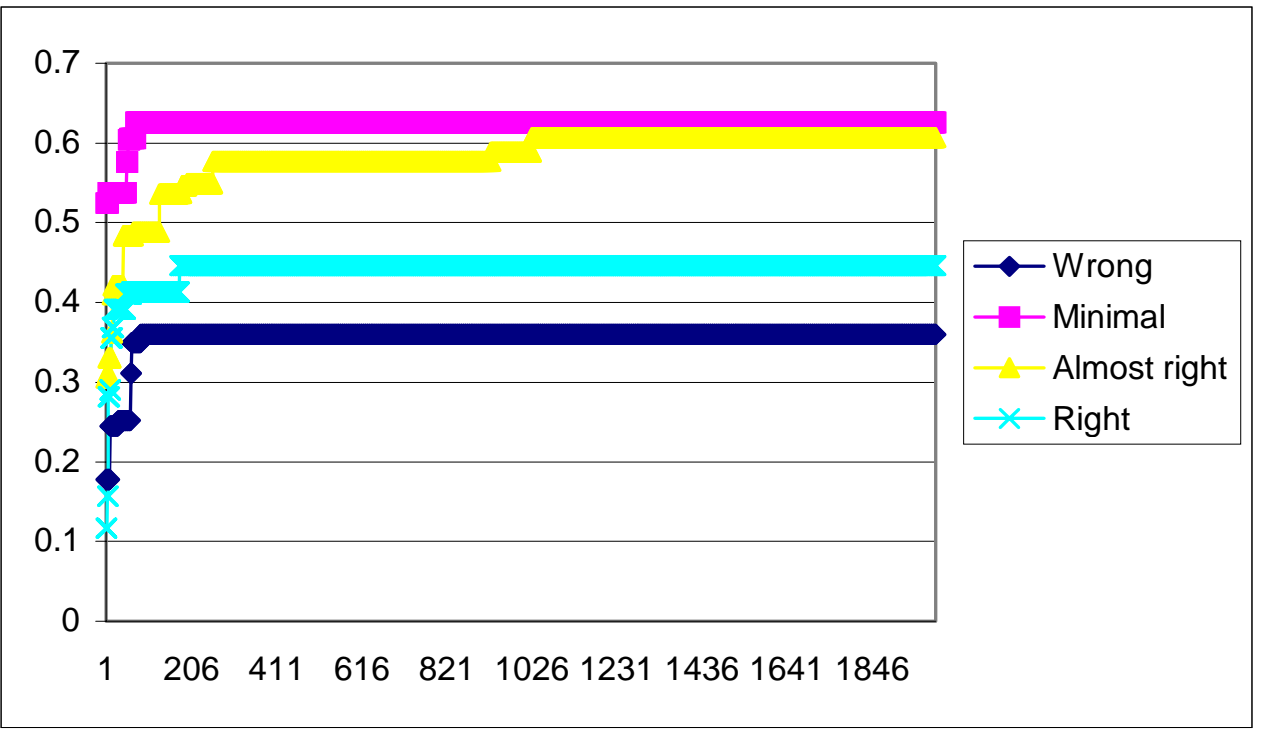

Figure 14: Interacton of Cognition and Control

(Average profit over 200 repetitions)

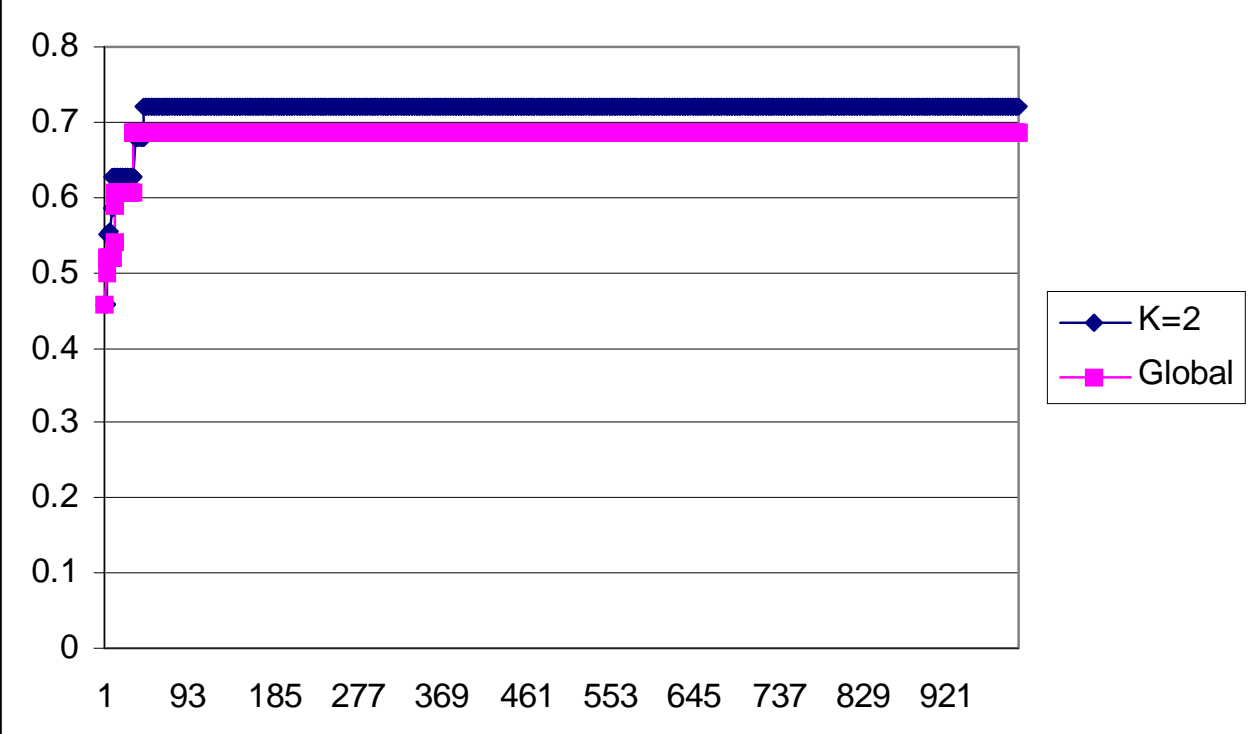

